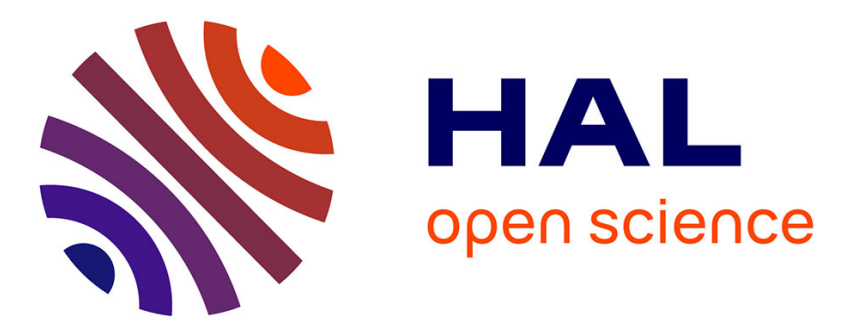

\title{
The rheology and thermal history of Mars revealed by the orbital evolution of Phobos
}

\author{
Henri Samuel, Philippe Lognonné, Mark P Panning, Valéry Lainey
}

\section{To cite this version:}

Henri Samuel, Philippe Lognonné, Mark P Panning, Valéry Lainey. The rheology and thermal history of Mars revealed by the orbital evolution of Phobos. Nature, 2019, 569 (7757), pp.523-527. 10.1038/s41586-019-1202-7 . hal-02380378

\section{HAL Id: hal-02380378 \\ https://hal.science/hal-02380378}

Submitted on 28 Nov 2019

HAL is a multi-disciplinary open access archive for the deposit and dissemination of scientific research documents, whether they are published or not. The documents may come from teaching and research institutions in France or abroad, or from public or private research centers.
L'archive ouverte pluridisciplinaire HAL, est destinée au dépôt et à la diffusion de documents scientifiques de niveau recherche, publiés ou non, émanant des établissements d'enseignement et de recherche français ou étrangers, des laboratoires publics ou privés. 


\title{
The rheology and thermal history of Mars revealed by the orbital evolution of Phobos
}

\author{
Henri Samuel $^{1}$, Philippe Lognonné ${ }^{1}$, Mark Panning ${ }^{2}$, Valéry Lainey ${ }^{2}$
}

1. Institut de Physique du Globe de Paris, CNRS, Université Sorbonne Paris Cité, Paris, France

2. Jet Propulsion Laboratory, California Institute of Technology, Pasadena, CA, USA

Preprint version ( Print version available at: www.nature.com/articles/s41586-019-1202-7 )

Reference: Nature 569, 523527 (2019), doi: 10.1038/s41586-019-1202-7

The evolution and internal structure of Mars are, by comparison to its present-day surface, poorly known -although evidence of recent volcanic activity ${ }^{1}$ suggests that its deep interior remains hot and convectively cooling. The cooling rate of Mars is related to its early thermal state and to its rheology, which determines its ability to deform and to dynamically evolve ${ }^{2}$. Attempts to reconstruct the dynamic history of Mars and reveal its present-day structure, by combining the study of thermal evolution with surface observations, are limited by the interplay between several key quantitiesincluding temperature, composition and rheology. Here we show that by considering Phobos (the closest satellite of Mars) the orbital evolution of which is governed by the thermochemical history of Mars, through tidal interactions we can gain insight into the thermal history and rheology of the planet. We investigated the longterm evolution of the main envelopes of Mars; these comprise a liquid metallic core that is overlain by a homogeneous silicate convecting mantle underneath an evolving heterogeneous lithospheric lid that includes a crust enriched in radiogenic elements. By exploiting the relationship between Mars and Phobos within an established in situ scenario for the early origin of the moons of Mars $^{3}$, we find that initially Mars was moderately hotter (100 to 200 kelvin) than it is today, and that its mantle sluggishly deforms in the dislocation creep regime. This corresponds to a reference viscosity of $10^{\mathbf{2 2 . 2}} \pm 0.5$ pascal seconds ( \pm denotes minimum and maximum range limits throughout), and to a moderately to relatively weak intrinsic sensitivity of viscosity to temperature and pressure. Our approach predicts a present-day average crustal thickness of $\mathbf{4 0} \pm \mathbf{2 5}$ kilometres and a surface heat flow of $\mathbf{2 0} \pm \mathbf{1}$ milliwatts per square metre. We show that combining these predictions with data from future and ongoing space missions - such as InSight - could reduce uncertainties in Martian thermal and rheological histories, and help to uncover the origin of Phobos.

To investigate the cooling history of Mars, we modelled its thermal evolution (see Supplementary Information, section $\mathrm{S} 1$, for details of the modelling approach) within a plausible range of various governing parameters: the initial core $\left(T c_{0}\right)$ and mantle $\left(T m_{0}\right)$ convective temperatures; the viscous rheology of the Martian mantle; the effective activation energy $\left(E^{*}\right)$ and volume $\left(V^{*}\right)$ of the mantle, which determine the sensitivity of viscosity to temperature and pressure; and the 
value of viscosity of the mantle $\left(\eta_{0}\right)$ at a reference temperature and pressure (see Supplementary Information, section S4, for details of the parameter space that we considered). Similar to many other planetary bodies, Mars behaves as a thermal engine. The initial thermal state of a planet is a proxy for the amount of energy that the planet has to evacuate, and the rheology of a planet controls the efficiency at which heat can be transferred within the planet and eventually lost to space. These two families of governing parameters are, however, not independent of each othermainly because the rheology of rocky planets is a strong function of temperature ${ }^{4}$ (Supplementary Information, section S1). This interdependency naturally leads to trade-offs: identical thermal states for present-day Mars can be obtained from different mantle rheologies (Fig. 1a, b). Dependencies such as these do not allow thermal and rheological quantities to be uniquely defined ${ }^{5,6}$, and further constraints that have different sensitivities to the governing parameters are required to remove these trade-offs. Unfortunately, eliminating these trade-offs by constraining the rheology of the Martian mantle from observations or experiments is difficult. On Earth, studies of post-glacial rebound enable estimates of the viscosity of the upper mantle ${ }^{7}$. These studies are not possible on Mars because such a record does not exist. Another way of inferring mantle- rock viscosity is by performing deformation experiments at high temperature and pressure ${ }^{4}$. However, these experiments are conducted on very small samples that are deformed at rates that are orders of magnitude above the strain rate that is imposed by convective motions ${ }^{8}$. These measurements therefore require considerable extrapolations, which are themselves not free of uncertainties. Multi-scale modelling is a tractable approach to determining the rheology of polycrystalline aggregates, although it requires parameter adjustment to match experimental observations ${ }^{9}$. Another substantial source of uncertainty derives from the influences of the chemical composition of the rocks in terms of major and minor elements ${ }^{4,10}$. This is particularly true for Mars, for which the only known samples that are currently available are thought to be the shergottite-nakhlite-chassignite (SNC) meteorites ${ }^{11}$. Consequently, the mantle rheology of Mars is both the most-important and the least- constrained physical parameter that controls its thermochemical evolution.

To better separate out the influences of temperature and rheology on the thermochemical evolution of Mars, we considered the coupled orbital evolution of its closest satellite, Phobos. Thermalorbital coupling results from tidal interactions between Mars and its satellite. A satellite exerts a deforming gravitational force that creates a tidal bulge at the surface of the orbited body. If the planet is attenuating (that is, if it does not behave as a purely elastic medium), the bulge is not aligned in the direction of the planet and the satellite but instead forms at an angle. On Earth, attenuation owing to tidal interaction mostly occurs through friction along the shorelines and at the bottom of the oceans. Because the palaeo-bathymetry of Earth is unknown, using the long- term orbital evolution of the Moon to constrain the history of the Earth is not possible. However, on Mars dissipation mostly occurs through viscous deformation within its interior although the influence of an early surface ocean could have a role (Supplementary Information, section 6.2). Such tidal interactions through viscous deformation are mainly governed by the ratio 

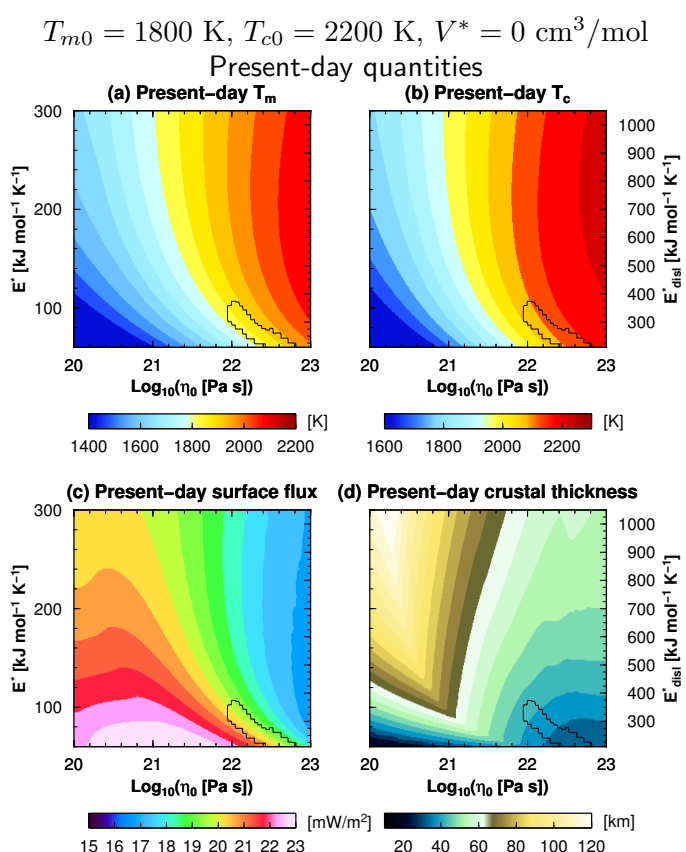

151617181920212223

Time-averaged and timing quantities
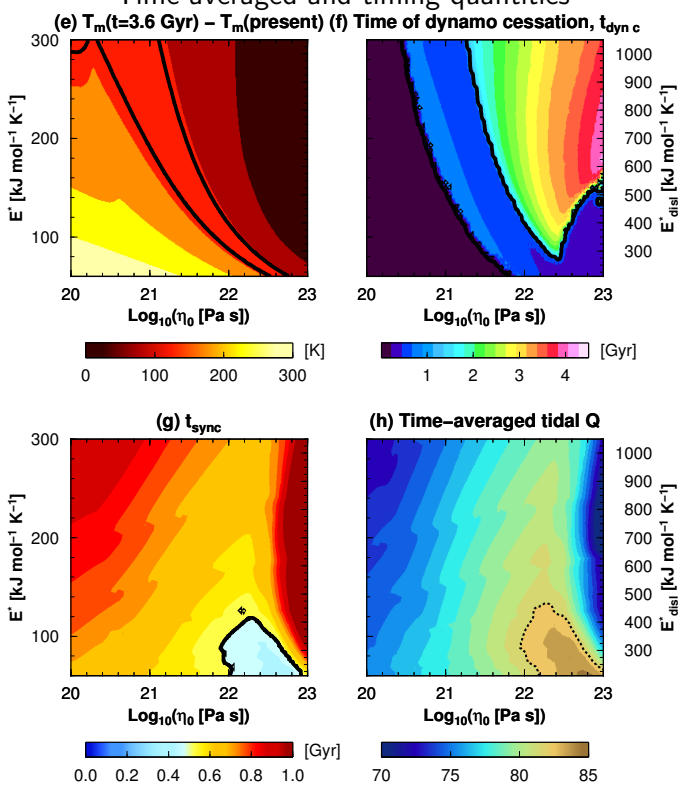

Figure 1: Exploration of Mars-Phobos' thermo-orbital evolution, for $T_{m 0}=1800 \mathbf{K}$, $T_{c 0}=2200 \mathbf{K}$, and $V^{*}=0 \mathbf{c m}^{3} / \mathbf{m o l}$. | (a-d) Present-day quantities. (e-h) Time-averaged and timing quantities. Each panel represents 4096 thermo-chemical and orbital evolutions, with distinct mantle reference viscosity $\eta_{0}$, and effective $\left(E^{*}\right)$ or dislocation creep $\left(E_{\text {disl }}^{*}=E^{*} / 3.5^{28}\right)$ activation energies. Black curves in (e-f), and (h) delineate respectively critical values for: the Amazonian-Hesperian secular cooling (100-140 K), the solutions corresponding to an early Martian thermal dynamo, and the (backward-going) time at which Phobos reaches the synchronous orbit. The dotted curve in (h) represents a time-averaged $Q=82$. Black contours in (a-d) mark the solutions satisfying thermal, magnetic, and orbital constraints (see Fig. 2). 
of the degree-two Love number of Mars $\left(k_{2}\right)$ over its tidal quality factor $(Q)^{12}$. The degree-two Love number describes the reaction of the gravitational potential of Mars to the tidal forcing, and is weakly sensitive to temperature within the solid mantle. By contrast, the tidal quality factor which measures the tidal dissipation within Mars - is inversely proportional to the tidal angle and is known to be strongly temperature-dependent ${ }^{13}$, according to an Ahrrenius relationship for a given pressure and frequency of $Q \sim \exp (b / T)$, in which $T$ is the mantle temperature and $b>1$ is a constant. A hotter mantle implies a lower $Q$, and thus a stronger tidal dissipation within Mars. A stronger tidal dissipation of a planetary body yields a faster change in the values of the orbital evolution parameters of its satellite. In the simplest case in which $Q$ would remain constant (or the temperature would not evolve with time) and the orbital eccentricities of Phobos were small, the evolution of the semi-major axis distance for the MarsPhobos system $(a)$ is a simple timedecreasing function of the ratio $k_{2} / Q$ :

$$
a=\left[-c t \frac{k_{2}}{Q}+a(t=0)^{13 / 2}\right]^{2 / 13},
$$

in which $c$ is a positive constant, and $t$ is time ${ }^{12}$. Therefore, the lower the value of $Q$ the faster Phobos converges towards the surface of Mars, as time increases. Although, in reality, the influence of eccentricity and the time-dependence of $Q$ cannot be neglected (Supplementary Information, sections S2, S3), the above reasoning remains qualitatively correct. Phobos, unlike its companion satellite Deimos, spirals in towards the surface of Mars. This difference between the two satellites results from Phobos being located within the synchronous limit (about 6.03 Martian radii) at which the angular velocity of the satellite exactly equals the spin of the planet, whereas Deimos is located beyond the synchronous limit. The origin of Phobos and Deimos remains debated and several scenarios advocate for an in situ origin, such as the satellites being the result of an early impact on Mars ${ }^{3,14,15}$ or the consequence of a disruption of a more-ancient moon ${ }^{14,16}$. An external origin has also been proposed, in which the Martian moons are suggested to result from asteroid capture during the presence of an early dense atmosphere ${ }^{17,18}$; this scenario, however, cannot explain the current small inclination of Phobos and Deimos ${ }^{16,19}$. For the in situ scenarios, the emplacement of Phobos within a time window of 0-500 million years (Myr) is commonly accepted, with the exception of a recent proposal that suggests the progressive accretion of debris from a giant impact ${ }^{20}$. We did not consider this possibility because an accretion scenario such as this would only take place within the synchronous orbit and would not, therefore, explain the origin of Deimos ${ }^{21}$. In addition, regardless of the scenario for the origin of Phobos, its present-day location ( $a=2.76$ Mars radii) within the synchronous radius implies that this satellite was emplaced at a distance from Mars that lies between its current location and the synchronous limit. We shall see that such a consideration for orbital evolution although simple exhibits a sensitivity to thermal and rheological parameters of Mars that is different from more-commonly used constraints. This makes orbital evolution a powerful tool, when combined with other constraints, for inferring the rheology and the thermal history of Mars.

We considered the following three constraints to reduce the solution space for plausible Mar- 
tian thermal evolution: (1) the mantle total secular cooling during the Amazonian and Hesperian periods (about 3.6 billion years (Gyr) ago until present), which is estimated to lie within 100-140 $\mathrm{K}$ on the basis of petrological considerations ${ }^{22}$ (Fig. 1e); (2) the presence of an internally induced magnetic field during the first billion years of the evolution of Mars ${ }^{23}$ (Fig. 1f) (see Supplementary Information, section S5, for details); and (3) the semi-major axis of the trajectory of Phobos around Mars must be located well within the synchronous radius during the past four billion years of orbital evolution. This last constraint can be reformulated as the time $\left(t_{\text {sync }}\right)$ at which Phobos approaches the synchronous radius being less than $500 \mathrm{Myr}$ (when tracing the evolution of Phobos backwards in time). This corresponds to the upper time limit that we considered for the formation of Phobos (Fig. 1g) (see Supplementary Information, sections S2, S4, for details). This limit approximately corresponds to a time-averaged value of $Q=82$ (Fig. 1h), which is only moderately below the present-day value of 95 (assuming a $Q / k_{2}$ ratio $=559^{24}$, and a present-day value of $k_{2}=0.169^{25}$ ). This indicates that orbital evolution constraints are very sensitive to the thermal history of Mars, as $Q$ is dependent on the thermal state. These present-day and time-averaged values of the tidal quality factor are considerably smaller than the present-day values inferred for the Earth $^{26}$. This suggests that at present the convecting mantle of Mars would be hotter than that of the Earth. Indeed, active plate tectonics on Earth would yield more-efficient heat loss over geological timescale, as compared to stagnant lid convection on Mars ${ }^{27}$ (see Supplementary Information, section S6.1). Alternatively, rheological differences between the Earth and Mars could have their origins in differences in the major- or minor-element composition of their mantles ${ }^{4,10}$.

Each of the constraints that we considered defines a reduced domain within the solution space (activation energy and reference viscosity) that is displayed in Fig. 2a-c, for a given set of initial mantle and core temperatures and given mantle activation volume. The area that is common to the three individual constraints points to the reduced matching set of rheological parameters (Fig. 2d), the contour of which is shown in Fig. 1a-d. The trade-offs between the thermal state and mantle rheology of Mars are clearly visible in Fig. 2, b, in which the solutions that satisfy both secular cooling and magnetic constraints are superimposed; this leaves a large space of acceptable solutions with very distinct rheologies. However, the additional consideration of the orbital evolution of Phobos largely removes the trade-offs, because the associated domain of acceptable solutions (Fig. 2c) only partially superimposes on the solutions defined by thermal and magnetic constraints. This illustrates the benefit of using orbital constraints.

The exercise displayed in Fig. 1 and Fig. 2 can be reproduced for different values of initial Martian thermal states and activation volumes (that is, values for $T m_{0}, T c_{0}$ and $V^{*}$ ), within the plausible ranges that we considered (Supplementary Information, section S5).

Not all sets of $T m_{0}, T c_{0}$ and $V^{*}$ admit a matching solution. As shown in Fig. 3a, effective activation volumes that are greater than or equal to $4 \mathrm{~cm}^{3} \mathrm{~mol}^{-1}$ do not allow for matching solu- 
$T_{m 0}=1800 \mathrm{~K}, T_{c 0}=2200 \mathrm{~K}, V_{\text {disl }}^{*}=0 \mathrm{~cm}^{3} / \mathrm{mol}$

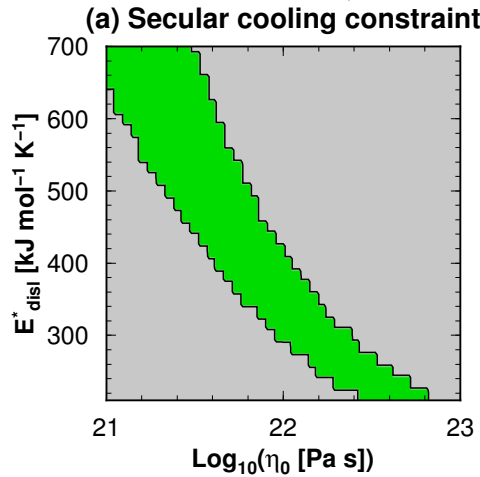

(b) Magnetic constraint

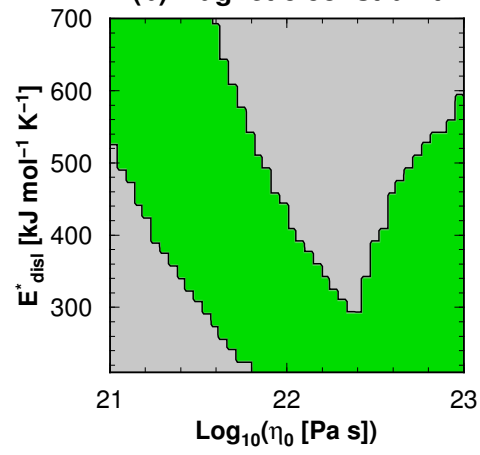

(c) Orbital constraint

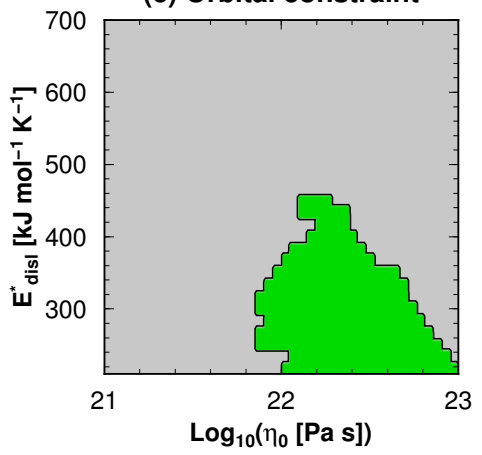

(d) Combined constraints

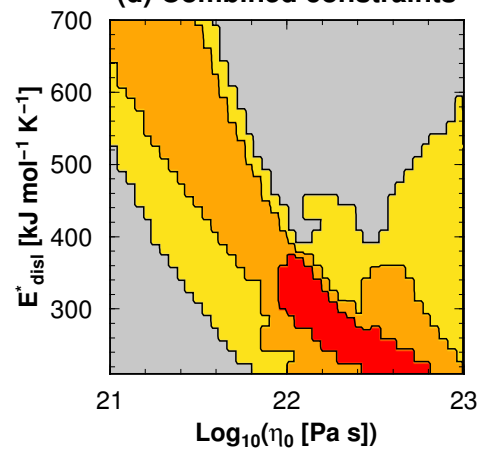

Matching index for individual constraints Non-matching solution

Matching constraint

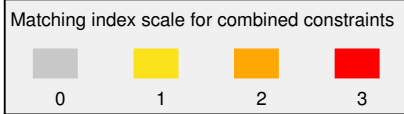

Figure 2: Thermal and orbital evolution constraints applied to the cases displayed in Fig. 1, assuming a Martian mantle deforming in the dislocation creep regime. | (a-c) Individual constraints. (d) combined constraints. The warmer colors indicate a higher matching index, while grey areas indicate thermal evolution that are not compatible any constraint considered. Red areas represent the mantle rheologies that satisfy all constraints. 
tions. Within the remaining space at $V *<\mathrm{cm}^{3} \mathrm{~mol}^{-1}$, the matching reference viscosity is about $5 \times 10^{21}-5 \times 10^{22}$ pascal seconds, and the effective activation energy is $E^{*} \cong 80 \pm 20 \mathrm{kJmol}^{-1}$. This range of relatively small values of effective activation energy is difficult to reconcile with the values inferred from experiments if the Martian mantle deforms in the diffusion creep regime ${ }^{4}$. However, if the Martian mantle deforms in the dislocation creep regime, the corresponding intrinsic activation energies and activation volume would be about $3.5 \times$ larger than the range of effective values mentioned above -a non-Newtonian, slowly deforming rock behaves similarly to a very viscous Newtonian fluid, with activation energies and activation volumes about $3.5 \times$ smaller that the corresponding intrinsic values ${ }^{28-30}$. This implies that the Martian mantle deforms in the dislocation creep regime, with an intrinsic activation energy of $E_{\text {disl }}^{*}=280 \pm 80 \mathrm{~kJ} / \mathrm{mol}$, and activation volume $V_{\text {disl }}^{*}<14 \mathrm{~cm}^{3} / \mathrm{mol}$.

Our approach also enables predictions to be made of the present-day values of several key measurable quantities, including the average surface heat flux of $20 \pm 1 \mathrm{~mW}$ per $\mathrm{m}^{2}$ and the average crustal thickness of $40 \mathrm{~km} \pm 25 \mathrm{~km}$. Both of these quantities slightly decrease with increasing activation volume (Fig. 3d-e). We have tested the influence of two potential additional early processes on our model results. The first of these is the effect of plate tectonics operating during the first 500 Myr of the thermochemical evolution of Mars (Supplementary Information, section S6.1). The influence of this is limited, and in essence moderately reduces the values of the matching intrinsic activation energies to $V_{\text {disl }}^{*}<10.5 \mathrm{~cm}^{3} / \mathrm{mol}$, the intrinsic activation energies to disl $E_{\text {disl }}^{*} \cong 250 \pm 40 \mathrm{~kJ} / \mathrm{mol}$ and the predicted range of present-day crustal thickness to $D_{c r} \cong 30 \pm 20 \mathrm{~km}$ (shown in Supplementary Fig. 8ae, respectively).

The second additional process that we tested was the effect of an early surface ocean (Supplementary Information, section S6.2). The presence of this ocean would further reduce the value of the matching intrinsic activation energies to $E_{\text {disl }}^{*} \cong 220 \pm 15 \mathrm{~kJ} / \mathrm{mol}$, and reduce the value of present-day crustal thickness to $33 \mathrm{~km} \pm 2 \mathrm{~km}$. In addition, the presence of an ocean would imply a very weak pressure dependence of the viscosity, or the complete absence of pressure-dependent viscosity (Supplementary Fig. 9a).

Overall, and despite uncertainties, these early potential complexities do not change the requirement for a 'sluggish Martian mantle that deforms under the dislocation creep mechanism. Additionally, our approach allows us to constrain the initial thermal state of Mars. Within the range of matching activation volumes, we found that the initial value for Tm is between $1,700 \mathrm{~K}$ and $1,900 \mathrm{~K}$ (Fig. 3f). However, this range gradually reduces to 1,700-1,800 K for the largest acceptable values of activation volume (Fig. $3 \mathrm{~g}-\mathrm{h}$ ). The matching range for $T c_{0}$ is related to the corresponding matching range for $T m_{0}$. This can approximately be described as $T_{c_{0}}=\min \left(T_{m_{0}}+200 \pm 100 \mathrm{~K}, 2300 \mathrm{~K}\right)$. The lower-end value underlines the fact that some initial positive temperature difference between the core and the mantle is required to drive an early dynamo. On the other hand, initial core tem- 

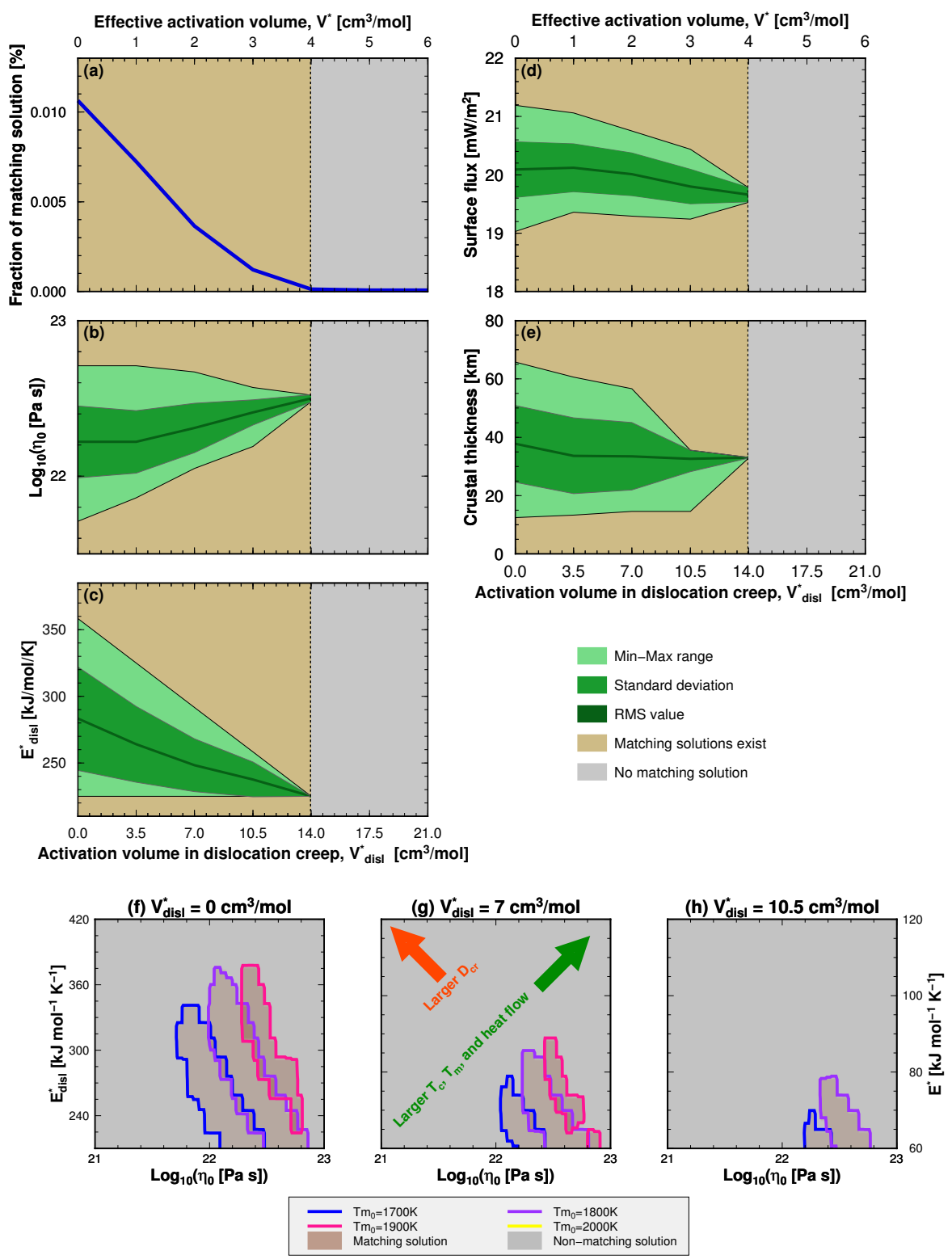

Figure 3: Results from the exploration of Mars-Phobos' thermal-orbital evolution. | (a-e) Statistics of present-day and time-averaged quantities of the matching solutions for the entire parameter space considered. (f-h) matching solutions as a function of the initial mantle thermal state, $T_{m 0}$, and mantle rheological parameters. Red and green arrows indicate the directions toward higher values of present-day crustal thickness, and surface heat flow, respectively. These directions concern the areas of matching solutions, and are unaffected by the lower and larger values of effective activation volume explored. 
peratures that are too hot yield large-scale deep mantle partial melting during the past four billion years of Martian thermochemical evolution. These melting events markedly lower the $Q$ value of Mars (Fig. 1h, Supplementary Information, section S1), which would force Phobos to have reached the synchronous limit well outside of the first 500 Myr of the history of Mars (Fig. 1g,). Within the matching area, $T m_{0}, T c_{0}$ and the surface heat flow increase when diagonally crossing the space ( $E^{*}$ and $\eta_{0}$ ) (Fig. 1a-c, along the direction of the green arrow in Fig. 3). Interestingly, the predicted present-day crustal thickness $\left(D_{c r}\right)$ does not vary along this direction (Fig. 1d), but is instead most sensitive to changes in $E^{*}$ and $\eta_{0}$ when moving perpendicular to this direction (Fig. 1d, red arrow in Fig. 3). When better-constrained by future observations, these markedly different sensitivities of heat flux and crustal thickness will allow us to refine estimates of the mantle rheology of Mars. Conversely, if the predictions of our model do not provide a reasonable match for future data, this could indicate that the thermal-orbital framework that we have considered (Supplementary Information) may itself have to be refined. This could also reveal that the convecting mantle of Mars is compositionally heterogeneous, and may have evolved as distinct convecting layers during a large part of the history of Mars ${ }^{31}$. A further possibility is that a mismatch between our predictions and future data could point towards an origin for Phobos ${ }^{20}$ that is different from the in situ scenario we considered.

The InSight mission aims to improve our understanding of the crustal thickness and surface heat flux of Mars using seismic recordings ${ }^{32}$ and heat flow measurements ${ }^{33}$, and will attempt to measure the gravimetric factor of the tide generated by Phobos. These data, when combined with the thermalorbital considerations discussed here, have the potential to considerably improve our understanding of the thermal history of Mars, as well as of its present-day structure and rheology. 
1. Hartmann, W. K. et al. Danielson, E., James, P., and Veverka, J. Evidence for recent volcanism on Mars from crater counts. Nature 397, 586-589 (1999).

2. Schubert, G., Turcotte, D., and Olson, P. Mantle convection in the Earth and Planets. Cambridge University Press, Cambridge (2001).

3. Rosenblatt, P. et al. Accretion of Phobos and Deimos in an extended debris disc stirred by transient moons. Nature Geosci. 9, 581-583 (2016).

4. Karato, S.-I. Deformation of Earth Materials: An Introduction to the Rheology of Solid Earth. Cambridge University Press (2008).

5. Hauck, S. A. and Phillips, R. J. Thermal and crustal evolution of Mars. J. Geophys. Res. 107 E7 (2002).

6. Grott, M. and Breuer, D. The evolution of the martian elastic lithosphere and implications for crustal and mantle rheology. Icarus 193, 503-515 (2008).

7. Mitrovica, J. X. Haskell [1935] revisited. J. Geophys. Res. 101, 555-569 (1996).

8. Cordier, P., Amodeo, J., and Carrez, P. Modelling the rheology of $\mathrm{MgO}$ under Earth's mantle pressure, temperature and strain rates. Nature 481, 177-180 (2012).

9. Raterron, P., et al. Multiscale modeling of upper mantle plasticity: From single-crystal rheology to multiphase aggregate deformation. Phys. Earth. Pl. Int. 228, 232-243 (2014).

10. Girard, J., Chen, J., Raterron, P., and Holyoke, C. W. Hydrolytic weakening of olivine at mantle pressure: Evidence of [100](010) slip system softening from single-crystal deformation experiments. Phys. Earth. Pl. Int. 216, 12-20 (2013).

11. Treiman, A. H., Gleason, J. D., and Bogard, D. The SNC meteorites are from Mars. Planet. Sp. Sci. 48, 1213-1230 (2000).

12. Kaula, W. M. Tidal Dissipation by Solid Friction and the Resulting Orbital Evolution. Rev. Geophys. Space Phys. 2, 661-685 (1964).

13. Jackson, I., Fitz Gerald, J. D., Faul, U. H., and Tan, B. H. Grain-size-sensitive seismic wave attenuation in polycrystalline olivine. J. Geophys. Res. 107:2360 (2002).

14. Rosenblatt, P. The origin of the Martian moons revisited. Astron. and Astrophys. Rev., 19, $1-26(2011)$.

15. Black, B. A. and Mittal, T. The demise of Phobos and development of a Martian ring system. Nature Geosci. 8, 913-917 (2015). 
16. Peale, S. J. Origin and evolution of the natural satellites. Ann. Rev. Astron. Astrophys. 37, 533-602 (1999).

17. Mignard, F. Evolution of the Martian satellites. Mon. Notices Royal Astron. Soc. 194, 365-379 (1981).

18. Burns, J. A. Contradictory clues as to the origin of the Martian moons. In M. George, editor, Mars, pages 1283-1301. University of Arizona Press (1992).

19. Szeto, A. M. K. Orbital evolution and origin of the Martian satellites. Icarus, 55, 133-168 (1983).

20. Hesselbrock, A. J. and Minton, D. A. An ongoing satellite-ring cycle of Mars and the origins of Phobos and Deimos. Nature Geosci. 10, 266-269 (2017).

21. Hyodo, R., Genda, H., Charnoz, S., and Rosenblatt, P. On the Impact Origin of Phobos and Deimos. I. Thermodynamic and Physical Aspects. ApJ 845:125, (2017).

22. Baratoux, D., Toplis, M., Monnereau, M., and Gasnault, O. Thermal history of Mars inferred from orbital geochemistry of volcanic provinces. Nature 472, 338-341 (2011).

23. Connerney, J. E. P., et al. Mars crustal magnetism. Sol. Sys. Res. 11, 1-32 (2004).

24. Zharkov, V. N. and Gudkova, T. V. Construction of Martian Interior Model. Sol. Sys. Res. 39, 343-373 (2005).

25. Konopliv, A. S., Park, R. S., and Folkner, W. M. An improved JPL Mars gravity field and orientation from Mars orbiter and lander tracking data. Icarus 274, 253-260 (2016).

26. Lognonné, P. and Mosser, B. Planetary Seismology. Surveys in Geophysics 14, 239-302 (1993).

27. Breuer, D. and Spohn, T. Early plate tectonics versus single-plate tectonics on Mars: Evidence from magnetic field history and crust evolution. J. Geophys. Res. 108:5072 (2003).

28. Christensen, U. R. Convection in a variable-viscosity fluid: Newtonian versus power-law rheology. Earth Planet. Sci. Lett. 64, 153-162 (1983).

29. Kiefer, W. S. and Li, Q. Water undersaturated mantle plume volcanism on present-day Mars. Met. Planet. Sci. 51, 1993-2010 (2016).

30. Thiriet, M., Michaut, C., Breuer, D., and Plesa, A.-C. Hemispheric Dichotomy in Lithosphere Thickness on Mars Caused by Differences in Crustal Structure and Composition. J. Geophy. Res.: Planets 123, 823-848 (2018).

31. Maurice, M. et al. Onset of solid-state mantle convection and mixing during magma ocean solidification. J. Geophy. Res.: Planets 122, 577-598 (2017). 
32. Lognonné, P., et al. SEIS: The Seismic Experiment for Internal Structure of InSight. Space Sci. Rev. 215:12, 1-170 (2019).

33. Spohn, T., et al., The Heat Flow and Physical Properties Package $\left(\mathrm{HP}^{3}\right)$ for the InSight Mission. Space Sci. Rev., 214:96, 1-33 (2018).

\section{Acknowledgments}

The authors thank S. Charnoz, A. Mocquet, and M. Thiriet for fruitful discussions. M. Thiriet is also gratefully acknowledged for providing her data for the benchmark comparison (Supplementary Fig. 2)). The software for the computation of the elastic Love number was kindly provided by J. Wahr. Numerical computations were performed on the S-CAPAD platform, IPGP, France. V.L.'s research was supported by an appointment to the NASA Postdoctoral Program at the NASA Jet Propulsion Laboratory, California Institute of Technology, administered by Universities Space Research Association under contract with NASA. P.L. and M.P. thank CIDER (NSF EAR-1135452) for providing the 2014 summer school environment at KITP UCSB during which an early version of this research was imagined and discussed. Figures were made with the Generic Mapping Tools (P. Wessel and W.H.F. Smith, EOS, Trans. AGU 76 (1995) 329). This is IPGP contribution number 4026 and InSight contribution number 76.

\section{Author contributions}

P.L. proposed the original idea. M.P. contributed to the original idea development. P.L. developed and tested the software for the computation of the Love number imaginary part. V.L. and H.S. developed and discussed the orbital evolution model. H.S. developed the thermo-chemical evolution and the seismic models, implemented the computer code for the coupled thermo-chemical and orbital evolution, performed the simulations and data analysis, produced the figures and wrote the paper. All authors discussed the paper and interpreted the results.

\section{Author Information}

Reprints and permissions information is available at www.nature.com/reprints. The authors declare no competing financial interests. Readers are welcome to comment on the online version of the paper. Correspondence and requests for materials should be addressed to H. S. (samuel@ipgp.fr).

* Code availability The code for computing the thermal and orbital evolutions is available on request from the corresponding author. 